\title{
Erratum to: A multicenter evaluation of a new post-processing method with depth-dependent collimator resolution applied to full-time and half-time acquisitions without and with simultaneously acquired attenuation correction
}

Carmelo V. Venero, MD, ${ }^{a}$ Gary V. Heller, MD, PhD, FACC, ${ }^{\mathrm{a}, \mathrm{b}}$

Timothy M. Bateman, MD, FACC, ${ }^{\mathrm{c}, \mathrm{d}}$ A. Iain McGhie, MD, FACC, ${ }^{\mathrm{c}}$

Alan W. Ahlberg, MA, ${ }^{a}$ Deborah Katten, RN, ${ }^{a}$ Staci A. Courter, MA, ${ }^{d}$

Robert J. Golub, MD, FACC, ${ }^{\mathrm{e} J a m e s} \mathrm{~A}$. Case, $\mathrm{PhD},{ }^{\mathrm{d}}$ and S. James Cullom, $\mathrm{PhD}^{\mathrm{d}}$

\section{ERRATUM TO: J NUCL CARDIOL}

DOI 10.1007/S12350-009-9106-9

This article was published with an incorrect Figure 2. The correct Figure 2 is shown below.
From Hartford Hospital, ${ }^{\mathrm{a}}$ Hartford, CT; University of Connecticut, Farmington, CT; Mid America Heart Institute and University of Missouri-Kansas City, ${ }^{\mathrm{c}}$ Kansas City, MO; Cardiovascular Imaging Technologies, ${ }^{\mathrm{d}}$ Kansas City, MO; and Cardiology Associates of Central Connecticut, ${ }^{\mathrm{e}}$ Wallingford, CT.

The online version of the original article can be found under doi:10.1007/s12350-009-9106-9.

Reprint requests: Gary V. Heller, MD, PhD, FACC, Hartford Hospital, 80 Seymour Street, Hartford, CT 06102; gheller@harthosp.org.

J Nucl Cardiol 2010;17:706

$1071-3581 / \$ 34.00$

Copyright $\odot 2010$ American Society of Nuclear Cardiology.

doi:10.1007/s12350-010-9269-4
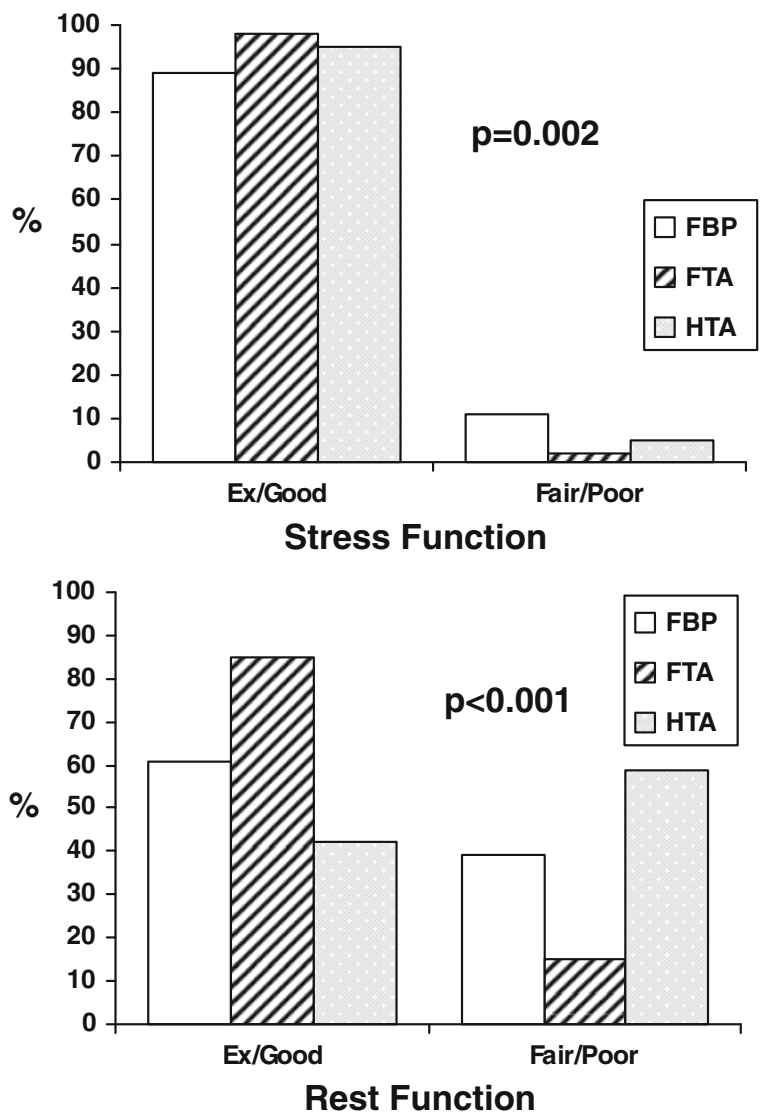

Figure 2. Image quality: stress gating (top), rest gating (bottom). FBP, Filtered backprojection; FTA, full-time Astonish; HTA, half-time Astonish. 